\title{
Satisfacción de los pacientes ante la atención odontológica recibida en un hospital en Lima
}

\author{
Satisfaction of patients to dental care received in a hospital in Lima \\ Jefferson Jonathan Lara-Alvarez ${ }^{1, a}$, Rocío Victoria Hermoza-Moquillaza ${ }^{1,2, \text { b, c }}$, CésarArellano-Sacramento ${ }^{\text {1, a, d }}$
}

\section{RESUMEN}

Objetivo: Determinar la satisfacción de los pacientes que recibieron un servicio odontológico en un hospital en Lima. Material y métodos: El estudio fue observacional, transversal y descriptivo. La muestra estuvo conformada por 702 pacientes. Se utilizó el instrumento SERVQUAL modificado para determinar la satisfacción del paciente. Se incluyeron pacientes mayores o igual a 18 años de edad con derecho de ser atendidos que acudieron al área odontológica. Resultados: La frecuencia de pacientes insatisfechos fue de 88,6\%, siendo 622 de 702 $(\mathrm{p}=0,000)$. La insatisfacción fue mayor en pacientes con una edad entre 35-49 años con un 34,5\% y en pacientes que acudían a diagnóstico con un 30,9\%; siendo en estos casos estadísticamente significativo. Conclusiones: La mayoría de pacientes que recibieron atención en el servicio odontológico estuvieron insatisfechos con la atención.

PALABRAS CLAVE: Satisfacción del paciente, calidad de la atención de salud, odontología estatal, Perú.

\section{SUMMARY}

Objective: To determine the satisfaction of patients who received a dental service in a hospital in Lima. Material and methods: The study was observational, cross-sectional and descriptive. The sample consisted of 702 patients. The modified SERVQUAL instrument was used to determine patient satisfaction. Patients older than or equal to 18 years of age with the right to be treated who attended the dental area were included. Results: The frequency of dissatisfied patients was $88.6 \%$, being 622 of $702(\mathrm{p}=0.000)$. Dissatisfaction was greater in patients aged between $35-49$ years with $34.5 \%$ and in patients who went to diagnosis with $30.9 \%$; being statistically significant in these cases. Conclusions: Most patients who received care in the dental service were dissatisfied with the care.

KEY WORDS: Patient satisfaction, quality of health care, state dentistry, Peru.

\footnotetext{
1 Universidad Norbert Wiener. Lima, Perú.

2 Dirección General de Medicamentos y Drogas, Ministerio de Salud. Lima, Perú.

a Cirujano Dentista.

b Químico Farmacéutico.

c Magister en Epidemiología Clínica.

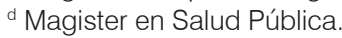




\section{INTRODUCCIÓN}

Es derecho de las personas ser atendidos en forma digna en los centros de salud; parte de esa forma de atención se refiere a la calidad en la atención (1). En el caso de Perú es misión del Ministerio de Salud (MINSA) el "proteger la dignidad personal, promoviendo la salud, previniendo las enfermedades y garantizando la atención integral de salud de todos los habitantes del país" (2); la satisfacción del usuario o paciente en sus demandas y expectativas es parte del estándar o indicador de calidad de atención(3).

La satisfacción del usuario respecto a la calidad de los servicios de salud es un tema complejo pues está relacionado a factores como edad, ingresos, educación, entre otros. Siendo actualmente un factor importante para generar planes de gestión en las instituciones de salud (4).

La forma de tratar la satisfacción del usuario ha cambiado con el tiempo. Primero se ligó a expresiones como calidad asistencial y rendimiento del servicio (evaluado directamente por el usuario). Luego fue concebida como una medida del resultado de la interacción profesional-paciente. Asimismo, se empezó a considerar su carácter multifactorial (5), relacionándolo con variables como sexo, edad, condición laboral, entre otras (6).

Un estudio en Perú (7) en el 2015 a nivel nacional encontró en usuarios de consulta externa un nivel de satisfacción del servicio del 73,7\% (el resto, 26,3\% "ni satisfecho ni insatisfecho" e "insatisfecho"); incluyeron usuarios entre 15 a 18 años y no muestran resultados del área de odontología. Otro estudio a nivel nacional muestra similares resultados (8).

La satisfacción de los usuarios puede mejorar con la aplicación de estrategias que simplifiquen y agilicen los procesos de atención en las instituciones de salud (9).

SERVQUAL es un instrumento para determinar el nivel de satisfacción con la calidad del servicio que se brinda al usuario de un servicio cualquiera (clientes, usuarios, pacientes, beneficiarios), siendo la comparación entre Expectativas (E) y Percepciones (P) del servicio una medida de la calidad del mismo (10).

SERQUAL es un instrumento que puede ser usado en diferentes ámbitos, como salud. El instrumento SERVQUAL ha sido modificado en idioma ingles para ser aplicado en labor hospitalaria (11). Conserva las cinco dimensiones originales pero solo considera 15 ítems y cinco de las siete alternativas de respuesta tipo Likert.

En el Perú SERVQUAL ha sido modificada $(12,13)$ para su aplicación hospitalaria; posteriormente SERVQUAL modificado por MINSA (SQM) (12) fue también modificado(14) para ser aplicada específicamente para hospitales del Seguro Social (EsSalud).

Para MINSA, la satisfacción del usuario externo es el grado de cumplimiento por parte de la organización de salud, respecto a las expectativas y percepciones del usuario en relación a los servicios que ésta le ofrece, $\mathrm{y}$, considera usuario externo, a la persona que acude a un establecimiento de salud para recibir una atención de salud de manera continua y con calidad, en el contexto de familia y comunidad (12).

La literatura ahonda información sobre satisfacción del usuario en diversas áreas de medicina excluyendo al área propiamente odontológica (15). Por ello, es necesario conocer el nivel de satisfacción de este usuario en particular para poder realizar los cambios o adecuaciones en la atención odontológica hospitalaria.

El objetivo del estudio fue determinar la satisfacción de los pacientes que recibieron un servicio odontológico en un hospital en Lima.

\section{MATERIAL Y MÉTODOS}

Estudio observacional, transversal, descriptivo. Se realizó el cálculo de muestra en base a una población de 1887 pacientes atendidos en un trimestre en tres áreas (operatoria, periodoncia, diagnóstico), utilizandose la fórmula para tamaño de muestra para proporciones con población finita, siendo el valor alfa de 0,05 , precisión de $3 \%$, proporción de $67 \%$ (12), resultando un tamaño de muestra de 629 , ajustado a pérdidas al $10 \%$ resultó en 699 , sin embargo, se consideró estudiar 702 .

Se incluyeron pacientes mayores o igual a 18 años de edad con derecho de ser atendidos que acudieron al área odontológica para su atención. Se excluyeron pacientes que manifestaban no saber leer ni escribir, pacientes que acudieron solo por emergencia, a 
acompañantes y/o tutores de los pacientes atendidos. De las cuatro escalas de SERVQUAL modificado por MINSA (SQM) (12), fue utilizado el referido a "servicio de consulta externa en establecimientos del primer nivel de atención". El SQM tiene por dimensiones: Fiabilidad (F), Capacidad de respuesta (CR), Seguridad (S), Empatía (E), Aspectos tangibles (T) (12).

El SQM consta de dos partes: Expectativa (Ex) o qué espera recibir, y, Percepción (Pe) o cómo recibió la atención, ambas con 22 ítems. Los valores de calificación de cada ítem son del 1 al 7 (a valor más bajo menor satisfacción) (12).

Se abordó en base a muestreo no probabilístico al paciente en las mañanas hasta completar 702 pacientes. Participaron de manera voluntaria, indicándoseles que su negativa a participar o sus respuestas no influirían en la atención brindada en el establecimiento. La recolección de datos fue realizada en 12 días laborables (12), teniendo como duración de 5 horas por día, en cada servicio.

La primera parte de la encuesta (Ex) se realizó en el pasillo de espera. Luego de la atención en el consultorio se les entrego nuevamente la prueba para que califiquen la segunda parte de la encuesta (Pe). El puntaje mínimo posible es 22 y el máximo 154 (tanto para Pe como Ex). Los valores resultantes de Pe y Ex se restan (Pe menos Ex), considerando como paciente Satisfecho a los valores positivos $(+)$ y cero, obtenidos por la diferencia entre Percepción y Expectativa. Siendo paciente Insatisfecho los valores negativos (-) de esta diferencia (12).

Según el instrumento se espera un "nivel de satisfacción global estándar” de más de $60 \%$ de Satisfacción para considerar logrados los estándares de atención en el servicio (12). Al evaluar individualmente los ítems, un porcentaje mayor a $60 \%$ de Insatisfacción se considera como "Por Mejorar", entre 40 a 60\% está "En Proceso" y menor de $40 \%$ es "Aceptable". Aquellos ítems que resulten mayores a $60 \%$ de Insatisfacción, son consideradas como "prioritarias para implementar acciones de mejora" o formular un "Proyecto de Mejora Continua" (12).

Para la creación de la base de datos, tablas de frecuencia y figuras se utilizó el programa Microsoft Excel. La parte estadística, tanto descriptiva como inferencial se realizó mediante el programa estadístico SPSS versión 22. El rango de edad se obtuvo en años basándonos en la categoría del titulado afiliar o derecho habiente (16) siendo estas 18-24, 25-34, 3549, 50-64, 65 a más. La variable sexo del paciente fue agrupada en las categorías biológicas hombre y mujer. La variable nivel educativo fue agrupada en las categorías Primaria, Secundaria, Superior técnico, Superior universitario, así como indica el MINSA (12); sin embargo, no se incluyó Analfabeto considerándosele un criterio de exclusión. El análisis de los datos fue expresado en porcentajes para cada criterio de evaluación de la calidad. Para la comparación en variables cualitativas se utilizó la prueba Chi cuadrado y prueba exacta de Fisher, tomándose en cuenta un valor alfa de 0,05 .

Se cumplió con las normas éticas exigidas internacionalmente para estudios observacionales. Se realizaron las solicitudes respectivas a la institución hospitalaria. El proyecto fue revisado y aceptado por la Escuela Académica Profesional de Odontología de la Universidad Privada Norbert Wiener, Lima, Perú. Se le consultó previamente al paciente sobre su deseo de participar de manera voluntaria (consentimiento). Fue realizada la codificación de los sujetos en la base de datos para mantener el anonimato.

\section{RESULTADOS}

La población encuestada estuvo conformada por 702 pacientes. La edad media fue de 42,8 años con una desviación estándar de 12,8 años, quienes mostraron las siguientes características: 385 pacientes $(54,8 \%)$ fueron hombres, 369 pacientes $(52,6 \%)$ tuvieron un nivel educativo superior técnico y 273 fueron pacientes entre 35-49 años(38,9\%) (tabla 1$)$.

La frecuencia de pacientes insatisfechos fue de $88,6 \%$, siendo 622 de $702(p=0,000)$.

La insatisfacción fue mayor en pacientes con una edad entre 35-49 años con un 34,5\%, en pacientes que acudían a diagnóstico con un $30,9 \%$; siendo en estos casos estadísticamente significativo. No se encontró diferencia estadística con sexo ni con nivel educativo (tabla 2). 
Tabla 1. Características de la población de estudio $(\mathrm{n}=702)$

\begin{tabular}{lll}
\hline $\begin{array}{l}\text { Variable } \\
\text { Sexo }\end{array}$ & $\mathbf{n}$ & $\mathbf{( \% )}$ \\
\hline Mujer & 317 & $(45,2)$ \\
Hombre & 385 & $(54,8)$
\end{tabular}

Edad

$\begin{array}{rcc}18-24 & 40 & (5,7) \\ 25-34 & 164 & (23,4) \\ 35-49 & 273 & (38,9) \\ 50-64 & 171 & (24,4) \\ 65 \text { a más } & 54 & (7,7)\end{array}$

Nivel educativo

Primaria $\quad 15 \quad(2,1)$

Secundaria $\quad 142 \quad(20,2)$

Superior técnico 369

Superior universitario 176

Tabla 2. Características de la población de estudio según satisfacción ( $n=702)$

\begin{tabular}{|c|c|c|c|c|c|}
\hline & \multicolumn{2}{|c|}{ Satisfecho } & \multicolumn{2}{|c|}{ Insatisfecho } & \multirow[b]{2}{*}{ Valor $\mathbf{p}$} \\
\hline & n & $\%$ & $\mathbf{n}$ & $\%$ & \\
\hline \multicolumn{6}{|l|}{ Sexo } \\
\hline Mujer & 42 & 6 & 275 & 39,2 & \\
\hline Hombre & 38 & 5,4 & 347 & 49,4 & $0,161 *$ \\
\hline \multicolumn{6}{|l|}{ Edad } \\
\hline $18-24$ & 13 & 1,9 & 27 & 3,8 & \\
\hline $25-34$ & 10 & 1,4 & 154 & 21,9 & \\
\hline $35-49$ & 31 & 4,4 & 242 & 34,5 & \\
\hline $50-64$ & 22 & 3,1 & 149 & 21,2 & \\
\hline 65 a más & 4 & 0,6 & 50 & 7,1 & $0,001 * *$ \\
\hline \multicolumn{6}{|l|}{ Nivel educativo } \\
\hline Primaria & 0 & 0 & 15 & 2,1 & \\
\hline Secundaria & 20 & 2,8 & 122 & 17,4 & \\
\hline Superior técnico & 38 & 5,4 & 331 & 47,2 & \\
\hline Superior universitario & 22 & 3,1 & 154 & 21,9 & $0,351 * *$ \\
\hline \multicolumn{6}{|l|}{ Especialidad } \\
\hline Operatoria & 20 & 2,8 & 194 & 27,6 & \\
\hline Periodoncia & 20 & 2,8 & 211 & 30,1 & \\
\hline Diagnóstico & 40 & 5,7 & 217 & 30,9 & $0,03^{*}$ \\
\hline
\end{tabular}

Chi cuadrado*

Prueba exacta de Fisher** 
Tabla 3. Frecuencia de satisfacción según dimensiones (Expectativa*) ( $n=702)^{*}$ Se muestra los ítems de Expectativa como referencia; los valores (\%) corresponden al resultado de la diferencia Percepción menos Expectativa.

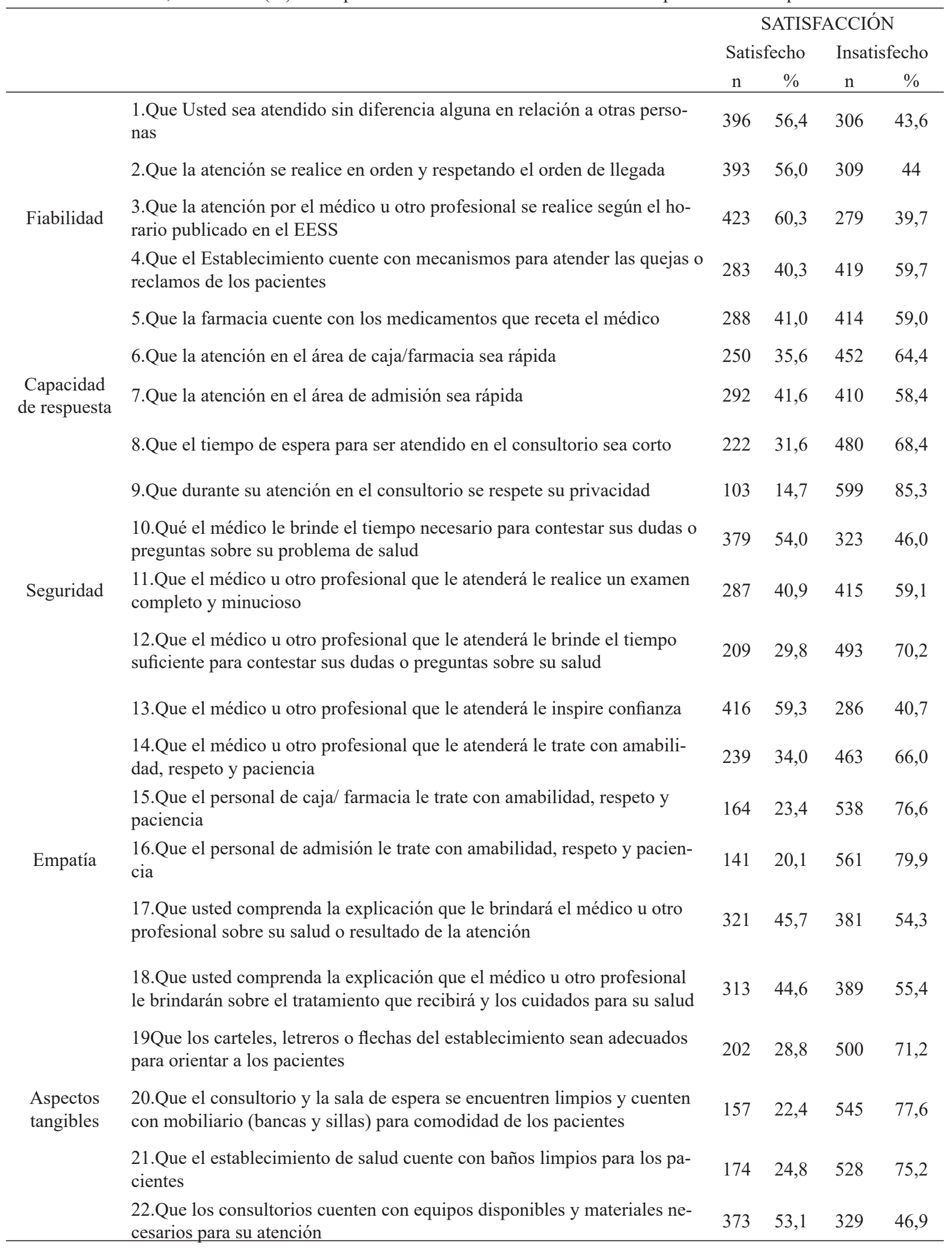


Con respecto al porcentaje de resultados de satisfacción: un ítem resultó "Aceptable", once "En Proceso" y diez "Por mejorar". Los ítems correspondientes a $6,8,9,12,14-16,19-21$ obtuvieron porcentajes de insatisfacción consideradas como "prioritarias para implementar acciones de mejora" o "formular Proyecto de Mejora Continua" (tabla 3).
La frecuencia de insatisfacción en la dimensión fiabilidad fue de $49.20 \%$, en capacidad de respuesta: $69,12 \%$, en seguridad: $54,02 \%$, en empatía: $66,44 \%$, en aspectos tangibles: $67,74 \%$ (gráfico 1).

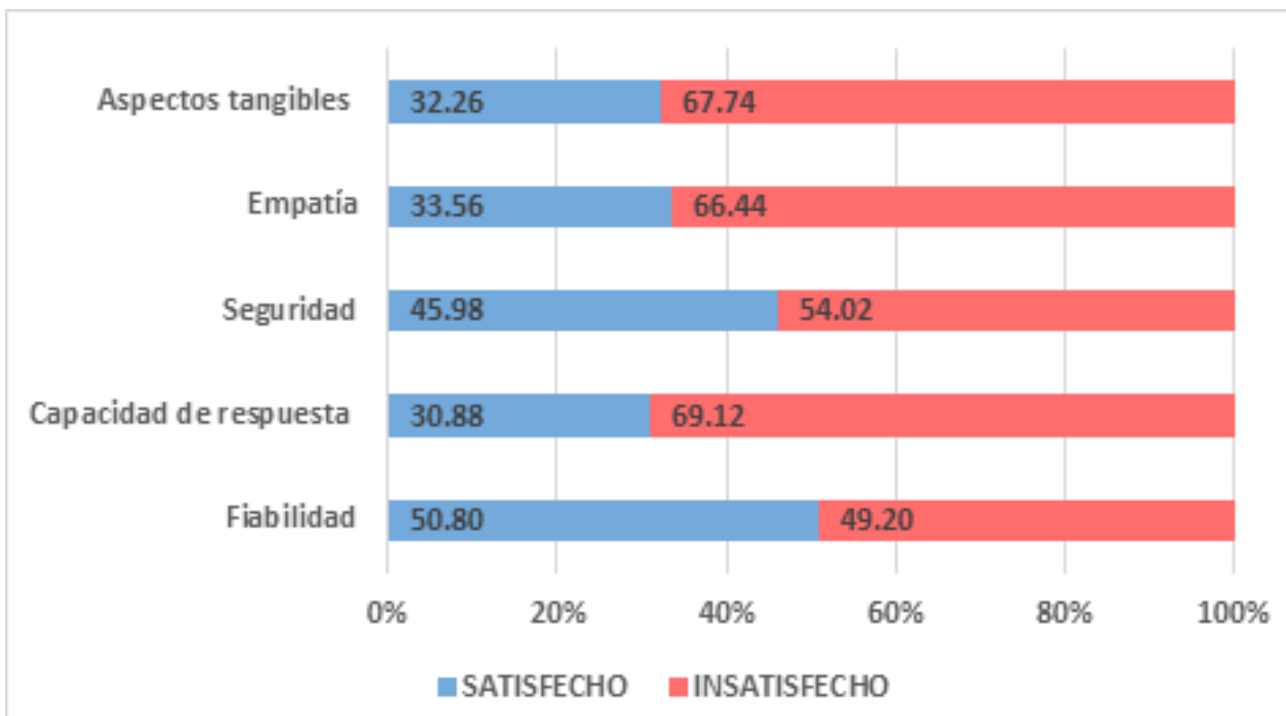

Gráfico 1. Frecuencia de satisfacción según dimensiones

\section{DISCUSIÓN}

No encontramos estudios dirigidos a conocer la satisfacción del paciente en el área de odontología hospitalaria que utilicen el mismo instrumento SERVQUAL modificado por MINSA. Por ello, se consideró realizar las comparaciones con estudios que utilicen instrumentos similares en servicios odontológicos hospitalarios estatales (sea o no del seguro social).

Encontramos en la literatura científica pocos estudios sobre satisfacción en la atención de pacientes odontológicos con puntos de corte para realizar comparaciones (17-19). Es necesario mencionar que, por razones de antigüedad, y de dudas sobre el instrumento utilizado, no incluimos algunos estudios para esta discusión.

Un estudio realizado en ocho municipios con Centros de Especialidades Odontológicas (CEO) de Bra- sil, utilizando el Questionário para Avaliação da Satisfação dos Usuários com Serviços de Saúde Bucal (QASSaB) encontró que la mayoría de los pacientes $(86,7 \%)$ estuvieron satisfechos con el servicio odontológico; el 10,2\% poco satisfecho y el 3,1\% muy satisfecho; mencionan que la variable sexo tuvo significancia estadística (no menciona el porcentaje). En el presente estudio no se encontró diferencia significativa en la variable sexo (17).

Una investigación en Perú realizada en pacientes odontológicos del seguro integral de salud, menciona encontrar un 5\% (6 de 120) de insatisfacción en la atención; no hace mayor mención del tipo de instrumento SERVQUAL utilizado (modificado a 18 ítems) (19). La presente investigación encontró valores mayores de insatisfacción.

En México, un estudio en pacientes de 30 módulos de salud bucal, menciona que el 88\% (434 de 493) 
reportó estar satisfecho con la atención del servicio dental (19), midieron la satisfacción con una única pregunta, mencionan la capacitación a los encuestadores y prueba piloto, pero no el procedimiento, la presente investigación encontró valores menores de satisfacción.

El presente estudio muestra que la institución hospitalaria no llegó al "nivel de satisfacción global estándar" requerido, siendo consideradas como "prioritarias para implementar acciones de mejora" o formular un "Proyecto de Mejora Continua".

Se concluye que la mayoría de pacientes atendidos en el área odontológica del hospital en Lima- Perú están insatisfechos con la atención. El porcentaje de insatisfacción fue mayor al $60 \%$ en las dimensiones Capacidad de respuesta (CR), Empatía (E), Aspectos tangibles $(\mathrm{T})$; además se encontró una diferencia de la variable edad y especialidad con respecto a satisfacción en la atención. No se encontró diferencia con sexo ni con nivel educativo.

Los resultados mostrados, nos pueden llevar a realizar cambios en los procesos de atención odontológica en la respectiva institución; el generalizar los resultados a instituciones similares a ésta y, como consecuencia, la mejora de la atención odontológica hospitalaria en el país, según MINSA, este indicador es recomendable que se mida como mínimo una vez al año (12).

La limitación del estudio es no poder ser comparado con otros estudios que hayan utilizado el mismo instrumento enfocados a la satisfacción del paciente odontológico. Otra limitación fue el corto periodo de tiempo estudiado, por ello, los resultados pueden no ser representativos de la población de usuarios anual; su fortaleza es mostrar resultados de insatisfacción utilizando un instrumento validado (12) por la entidad estatal encargada de ejercer la rectoría del Sector Salud en Perú, el Ministerio de Salud del Perú (12).

Recomendamos realizar estudios sobre satisfacción del paciente en diferentes centros de atención odontológica de las fuerzas armadas y civiles (sea o no seguridad social) comparando, en lo posible, entre instituciones y áreas odontológicas. Realizar investigaciones que comparen la satisfacción del familiar o acompañante y el paciente; así como, incluir a pacientes analfabetos.
Los datos de este estudio forman parte de la tesis de Lara Alvarez, Jefferson Jonathan para obtener el título de Cirujano Dentista en la Universidad Privada Norbert Wiener, Lima-Perú.

Declaración de Conflictos de Intereses: ninguno Fuente de financiamiento: Este trabajo de investigación no ha recibido ningún tipo de apoyo financiero específico de instituciones públicas, privadas o sin ánimo de lucro.

\section{Correspondencia:}

Cesar Arellano Sacramento

Correo Electrónico: cesar.arellano@uwiener.edu.pe

\section{REFERENCIAS BIBLIOGRAFICAS}

1. World Health Organization .Salud y derechos humanos. Ginebra: World Health Organization; 2017. (Citado 23 de junio del 2020) Disponible en: http://www.who.int/mediacentre/factsheets/ fs323/es/

2. Ministerio de Salud. Misión y visión. Lima: Ministerio de Salud;2020. (Citado 23 de junio del 2020) Disponible en: https://www.minsa.gob.pe/ transparencia/index.asp?op=103

3. Ministerio de Salud. Reglamento de establecimientos de salud y servicios médico de apoyo D.S. 013-2006-SA. Lima: Diario Oficial El Peruano; 25 de junio de 2006-

4. Numpaque-Pacabaque A, Rocha-Buelvas A. Modelos SERVQUAL y SERVQHOS para la evaluación de calidad de los servicios de salud. Rev Fac Med. 2016;64(4):715-20. Doi: doi. org/10.15446/revfacmed.v64n4.54839.

5. Cófreces P, Ofman S, Stefani D. La comunicación en la relación médico-paciente. Análisis de la literatura científica entre 1990 y 2010. Rev Comun Salud. 2014; 4:19-34.

6. Soto-Becerra P, Virú-Loza MA, Elorreaga OA, et al. Factores asociados a la calidad de la atención en población adulta afiliada a la Seguridad Social: El caso peruano. Rev Cuerpo Med HNAAA. 2020;13(1):14-25.

7. Ministerio de Salud. Encuesta Nacional de Satisfacción de Usuarios en Salud. Ministerio de Salud, ENSUSALUD; 2015.

8. Hernández-Vásquez A, Rojas-Roque C, Prado-Galbarro FJ, Bendezu-Quispe G. Satisfac- 
ción del usuario externo con la atención en establecimientos del Ministerio de Salud de Perú y sus factores asociados. Rev Peru Med Exp Salud Publica. 2019;36(4):620-8. doi: 10.17843/rpmesp.2019.364.4515.

9. Becerra-Canales B, Condori-Becerra A. Satisfacción de usuarios en hospitales públicos: experiencia del plan «Cero Colas» en Ica, Perú. Rev Peru Med Exp Salud Publica. 2019;36(4):65863. doi: 10.17843/rpmesp.2019.364.4299.

10. Parasuraman A, Zeithaml VA, Berry LL. SERVQUAL: A multiple- Item Scale for measuring consumer perceptions of service quality. Journal of Retailing. 1988;64(1):12-40.

11. Babakus E, Mangold WG: Adapting the SERVQUAL scale to hospital services:

an empirical investigation. Health Serv Res. 1992; 26(6): 767-86.

12. Ministerio de Salud. Guía Técnica para la Evaluación de la Satisfacción del Usuario Externo en los Establecimientos de Salud y Servicios Médicos de Apoyo, R.M. N 527 -

2011/MINSA/Ministerio de Salud. Lima: Ministerio de Salud; 2012.

13. Cabello E, Chirinos JL. Validación y aplicabilidad de encuestas SERVQUAL modificadas para medir la satisfacción de usuarios externos en servicios de salud. Rev Med Hered. 2012; 23(2):88-95.
14. Villar M, Ybañez Cuba E, Gutierrez Villafuerte C, Angulo-Bazán Y. Percepción de la calidad de los servicios de Medicina Complementaria del Seguro Social de Salud. Revista Peruana de Medicina Integrativa. 2016;1(3):27-33.

15. Numpaque-Pacabaque A, Rocha-Buelvas A. Modelos SERVQUAL y SERVQHOS para la evaluación de calidad de los servicios de salud. Rev Fac Med. 2016;64(4): 715-20.

16. EsSalud. Manual Institucional. Lima: EsSalud; 2008.

17. Kitamura ES, Bastos RR, Palma PV, Leite ICG. Avaliação da satisfação dos usuários dos Centros de Especialidades Odontológicas da macrorregião Sudeste de Minas Gerais, 2013. Epidemiol Serv Saude. 2016; 25(1):137-148.

18. Trejo CY. Nivel de satisfacción de pacientes adultos del Seguro Integral de Salud que acuden al Departamento de Odontoestomatología del Hospital Carlos Lanfranco la Hoz. Puente Piedra. Lima-Perú, 2016. Tesis de Grado. Lima: Universidad Privada Norbert Wiener; 2016.

19. Reyes-Fernández S, Paredes-Solís S, Legorreta-Soberanis J, Romero-Castro NS, Flores M, Andersson N. Satisfacción de usuarios con los servicios de salud bucal y factores asociados en Acapulco, México. Rev Cubana Estomatol. 2015;52(3):19-28.

Recibido: 20-12-19

Aceptado: 15-04-20 